Research Article

\title{
Measurement-Based Modal Analysis and Stability Prediction on Turn-Milling of Hollow Turbine Blade
}

\author{
Zhengcai Zhao $\mathbb{D}^{1,2}$ Junming Hou $\mathbb{D}^{1,3}$ and Yucan Fu ${ }^{1}{ }^{1}$ \\ ${ }^{1}$ College of Mechanical and Electrical Engineering, Nanjing University of Aeronautics and Astronautics, Nanjing 210016, China \\ ${ }^{2}$ Jiangsu Key Laboratory of Precision and Micro-Manufacturing Technology, Nanjing University of Aeronautics and Astronautics, \\ Nanjing 210016, China \\ ${ }^{3}$ Key Laboratory of Advanced Numerical Control Technology, Nanjing Institute of Technology, Nanjing 211167, China
}

Correspondence should be addressed to Zhengcai Zhao; zhengcaizhao@nuaa.edu.cn and Yucan Fu; yucanfu@nuaa.edu.cn

Received 24 June 2020; Revised 19 August 2020; Accepted 7 September 2020; Published 24 September 2020

Academic Editor: Paolo Pennacchi

Copyright (c) 2020 Zhengcai Zhao et al. This is an open access article distributed under the Creative Commons Attribution License, which permits unrestricted use, distribution, and reproduction in any medium, provided the original work is properly cited.

\begin{abstract}
Hollow blades with honeycomb structures are increasingly used in the turbine engines for reducing weight and saving costs. The hollow blade is a typical thin-walled structural part with low stiffness, the machining system of which is often unstable and likely to chatter. The most effective solution to avoid the machining chatter is to guide the hollow blade to be machined in a stable machining zone. This paper proposes a measurement-based approach for modal analysis and stability prediction of turn-milling hollow blade. The impact test was carried out to achieve the FRF curves on the hollow blade and the milling tool. An extremum method was employed to obtain an equivalent FRF curve, from which the modal parameters involving the natural frequency, damping ratio, and stiffness were computed. Afterwards, the semidiscretization method was used to draw a stability lobe diagram to predict the stability when turn-milling hollow blades. The experimental results confirm the feasibility of the predicted stability lobe diagram.
\end{abstract}

\section{Introduction}

Hollow blades with honeycomb structures (HCSs) are increasingly employed in the advanced turbine engines for lightweight and cost saving [1-3]. Superplastic forming (SPF)/diffusion bonding (DB) combined with numerical control machining (NCM) is the major solution to manufacture the hollow blades nowadays [4]. A near-net shape of the blade is formed by the processes of SPF and DB and then machined to its final shape by the NCM process. The hollow blade with HCSs is a typical thin-walled part, which has a curve surface and a weak stiffness. The setup of machining hollow blade with HCSs is complicated and multiaxis machining is often required (cf. Figure 1). The machining system of the hollow blade with HCSs is unstable and likely to vibrate strongly due to its weak stiffness [5]. Besides, the hollow blade with HCSs is often made of difficult-to-manufacturing titanium alloy, large cutting forces of which would intensify the vibration of the machining system. Self-excited vibration is one of the vibrations generated in the machining process of the hollow blade with HCSs. Self-excited vibration is well-known as the machining chatter, which is majorly caused by the unstable machining process. The machining chatter always results in some undesirable consequences such as poor surface finish and increased levels of tool wear [6-9]. The chatter occurring in the processing of the turbine blades with HCSs can even deteriorate the performance and life of the turbine engines. The most effective solution to avoid the chatter in machining of the hollow blade with HCSs is to make the machining process stable. The key to this solution is to find a stable machining zone and guide the process within this zone to achieve a stable output [10-12].

Dynamic parameters of the machining system are regularly analyzed and employed to predict the machining stability. Altintas and Budak [13] reported that the parameters of natural frequency, damping ratio, and stiffness can be used to express the dynamics of the machining system 


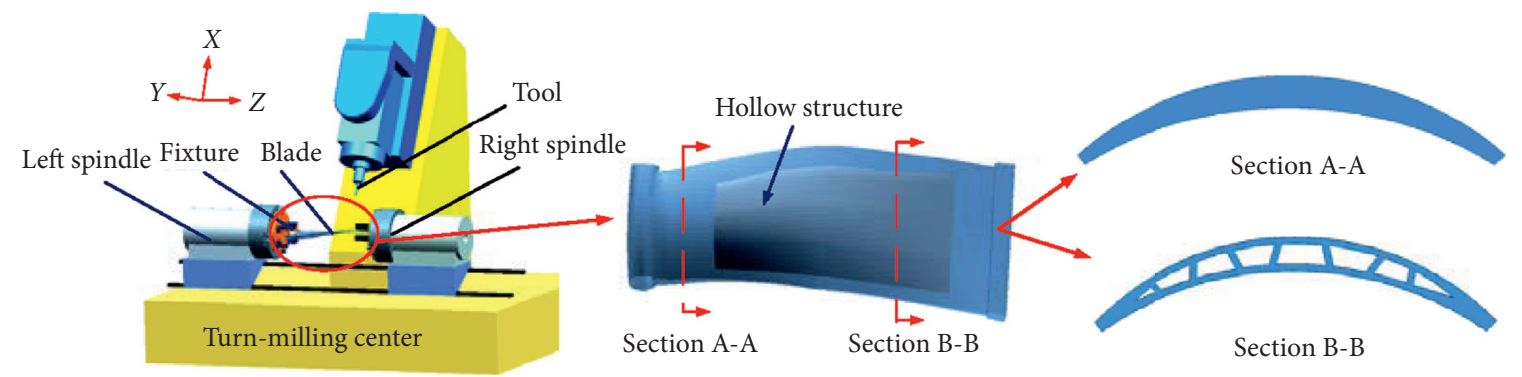

Figure 1: Turn-milling of the hollow blade with HCSs.

of the blade. The impact test and modal analysis are important analyzing methods for the dynamic parameters. The impact test is used to construct the relationship between the response displacement and the exciting force. This relationship is normally expressed in a formula which is called a transferring function. The Fourier transform of this transferring function is known as the frequency response function (FRF). The fitting methods are widely used to calculate the dynamic parameters based on FRF. Liu et al. [14] used a fitting method to achieve the dynamic parameters of the tooltip from FRF. Sun and Altintas [15] and Song et al. [16] build FRF of the cutting tool by impact test and identified the modal parameters using the fitting method. From [13-16], it is known that the fitting method is easily performed in computing the modal parameters. However, the fitting method always tries to obtain a mean curve of FRF which would dismiss the peaks of the original FRF curve. The response displacements of these peaks are larger than those of the fitting FRF curve. Thus, the computed modal parameters from the fitting FRF curve are incorrect which would result in a poor prediction of the machining system.

The stability of the machining system can be predicted based on the dynamic parameters, on which much previous work has been carried out. The semidiscretization method (SDM) is a commonly used method to predict the machining stability, which was firstly proposed by Altintas and Budak [13] and has been developed and improved furtherly by many scholars. The zero-order Fourier term of the dynamic parameters is used to approximate the variation of the cutting forces and predict the stability of the machining system by drawing a stability lobe diagram (SLD). Budak and Altintas [17] developed a stability prediction model to increase the predicting accuracy of the stability for machining the flexible workpiece. Insperger and Stepan [18] proposed an improved SDM and build a SLD for the periodic machining system. Henninger and Eberhard [19] presented a method of increasing the efficiency of SMD by accelerating the computation of a transition matrix. Since SDM has many applications in predicting the stability of the machining process, limited work on predicting the machining stability of hollow blades has been reported. Zhou et al. [20] established a 3-dimensional SLD by using a finite element method (FEM) for machining of the hollow blade. Although FEM has well-known advantages in the experimental methods, the accuracy of FEM is affected by the boundary conditions of the machining system, which is difficult to be in accordance with the actual machining conditions.
For the purpose of improving the system stability and avoiding the chatter in machining hollow blade with HCSs, this paper proposes a measurement-based approach for modal analysis and stability prediction of turn-milling hollow blade with HCSs. The impact test is carried out to achieve the FRF curves on the hollow blade and the cutting tool. An extremum method was employed to obtain an equivalent FRF curve, from which the modal parameters involving the natural frequency, damping ratio, and stiffness were computed. Afterwards, SDM was used to draw a SLD to predict the stability when machining hollow blades with HCSs. The paper is organized as follows. The setup of the impact test is presented in Section 2. Section 3 details the extremum method and an equivalent FRF curve is given. The stability prediction of machining hollow blade with HCSs is presented in Section 4, which has been validated experimentally. Conclusions are given in Section 5 finally.

\section{Setup of Impact Test}

An impact test was implemented to obtain the FRF curves of the milling tool and hollow blade, the setup of which is shown in Figure 2. The hollow blade is fixed on a turnmilling center typed Mazak 200 IV ST with the designed fixtures, which were clamped by the chucks of the machining tool. Ten regions (1) (10) are distributed on the tailing and leading edges of the hollow blade, on which the hammer knocked. An accelerometer typed PCB 356A32 with a sensitivity of $12.09996 \mathrm{mV} / \mathrm{m} \bullet \hat{s} 2$ was placed on the blade to inspect the response signals from each measured point, which was collected and analyzed by a Benstone Impaq Elite dynamic signal analyzer (cf. Figure 3 ). The sensitivity of the hammer was set as $2.5 \mathrm{mV} / \mathrm{N}$. The signals collected in the signal analyzer were in the time domain, which were transformed by the Fourier transfer to obtain the FRF curves in the frequency domain.

It can be seen that vibration marks are the manifestation of the displacements of tool and blade on the surface of the blade in the machining process. Considering that the larger contribution to the vibration marks of the blade surface is the normal displacement of the blade, the measuring direction of the accelerator and the exciting direction of the hammer are both set in this normal direction (cf. Figure 4). It should be noted that the axial direction of the milling tool has a certain angle $\beta$ with the tangential direction of this paper. 


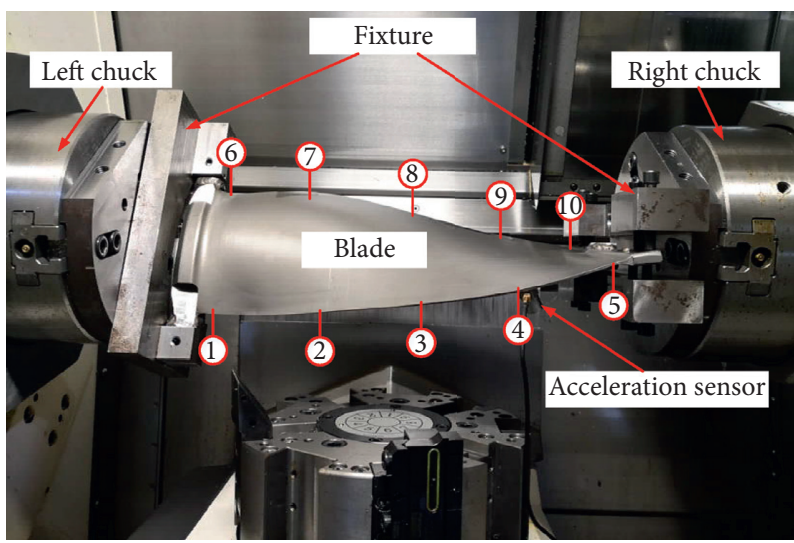

Figure 2: Setup of impact test.

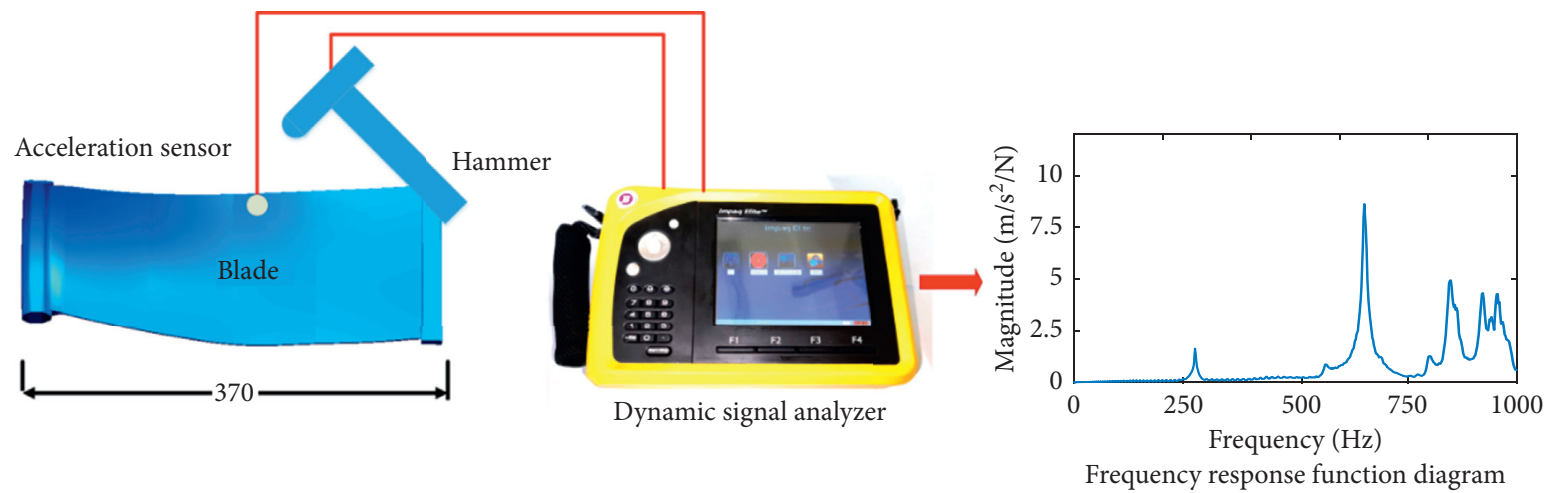

FIGURE 3: Measuring and analyzing the FRF curve.
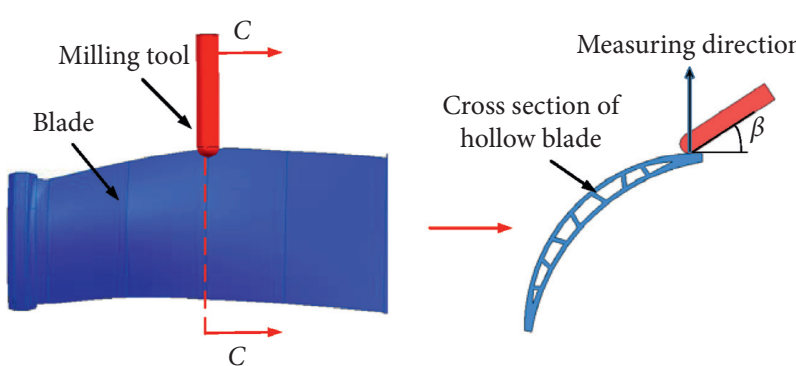

Figure 4: Schematic diagram of measuring directions.

\section{Modal Analysis on Machining System of Hollow Blade with HCSs}

The machining system of turn-milling hollow blades with HCSs consists of the hollow blade, the cutting tool, and the fixture. The vibration marks on the blade surface are mainly caused by the normal displacement of the blade. The system is simplified as a single-degree-of-freedom system and the displacement in the normal direction along the machined surface of the hollow blade is considered. The dynamic model of the machining system involving the hollow blade and the milling tool can be formulated as follows [21]:

$$
m \ddot{x}(t)+c \dot{x}(t)+k x(t)=F(t),
$$

where $\ddot{x}(t), \dot{x}(t), x(t)$ are the acceleration, velocity, and displacement, respectively. $m$ is the mass of the system, $k$ is the stiffness, $c$ is the damping, and $F$ is the exciting force of the system. This equation can also be expressed as

$$
\ddot{x}(t)+2 \varepsilon \omega_{n} \dot{x}(t)+\omega_{n}^{2} x(t)=\frac{\omega_{n}^{2}}{k} F(t),
$$

where the damping ratio $\varepsilon$ and the natural frequency $\omega_{n}$ are calculated as

$$
\begin{gathered}
\varepsilon=\frac{c}{2 \sqrt{k m}}, \\
\omega_{n}=\sqrt{\left(\frac{k}{m}\right)} .
\end{gathered}
$$

The harmonic force can be expressed as $F(t)=F_{0} e^{j \omega t}$, and the harmonic response is $x(t)=X e^{j(w t+\varnothing)}$ in equation (2). The equations can be transformed from the time domain to the frequency domain by using a Fourier transforming. By neglecting the effects of the initial conditions, equation (2) is transferred and expressed as follows:

$$
X e^{j(w t+\varnothing)}=\frac{\omega_{n}^{2}}{k} F_{0} e^{j w t}
$$

Thus, the FRF curve is expressed as 


$$
\Phi(\omega)=\frac{x(\omega)}{F(\omega)}=\frac{\omega_{n}^{2}}{k} \frac{1}{\omega_{n}^{2}+j 2 \varepsilon \omega_{n} \omega-\omega^{2}} .
$$

Then, the magnitude of the FRF is given by

$$
|\Phi(\omega)|=\left|\frac{x(\omega)}{F(\omega)}\right|=\left|\frac{\omega_{n}^{2}}{k} \frac{1}{\omega_{n}^{2}+j 2 \varepsilon \omega_{n} \omega-\omega^{2}}\right| .
$$

When the FRF is converted to the ratio of the acceleration to the force, the magnitude of the FRF is expressed as

$$
\begin{aligned}
|\Phi(\omega)| & =\left|\frac{\ddot{x}(\omega)}{F(\omega)}\right|=\left|\frac{\omega_{n}^{2}}{k} \frac{\omega^{2}}{\omega_{n}^{2}+j 2 \varepsilon \omega_{n} \omega-\omega^{2}}\right| \\
& =\frac{\omega_{n}^{2}}{k} \frac{\omega^{2}}{\sqrt{\left(\omega_{n}^{2}-\omega^{2}\right)^{2}+\left(2 \varepsilon \omega_{n} \omega\right)^{2}}} .
\end{aligned}
$$

The maximum magnitude of $\Phi(\omega)$ occurs at $\omega_{n}=\omega \sqrt{1-2 \varepsilon^{2}}$.

The machining modes dominate the dynamic response of the entire system, which contains the tool and workpiece systems. The transfer function is expressed as

$$
\Phi(\omega)=\frac{x(\omega)}{F(\omega)}=\Phi_{t}(\omega)+\Phi_{w}(\omega)
$$

where $\Phi_{t}(\omega)$ and $\Phi_{w}(\omega)$ are the transfer functions of the tool and workpiece systems, respectively.

The magnitude of FRF curves on regions (1)-(5) of the hollow blade (as Figure 2), which were measured by the impact tests, is presented in Figure 5. The FRF curves on regions (1)-(5), which are shown with the magnitudes, are expressed as P1-P5 and the range of the frequency is 0-1000 Hz. The magnitudes of FRF curves on regions (1)-(5) are quite different which is due to the difference of the dynamic parameters of different regions on the hollow blade. It can be seen from Figure 5 that the magnitude of P4 is slightly larger than that of other regions, and the magnitude of P1 curve is the smallest. In the same method, the FRF curves on regions (6)-(10), which are shown with the magnitudes, are presented as P6-P10 in Figure 6. It is shown that the FRF curves have similar trends and two peaks of the magnitude can be found in the range of $0-1000 \mathrm{~Hz}$. The natural frequencies corresponding to the peaks are approximately 300 and $600 \mathrm{~Hz}$, respectively.

Modal analysis is a common method used to obtain the dynamic parameters of the machining system. The dynamic parameters of different regions on the hollow blade can be calculated based on the magnitude of FRF curves. By using the previous dynamic model, the dynamic parameters involving the natural frequency, damping ratio, and stiffness were calculated based on the FRF curves in Figures 5 and 6. The calculated stiffness at ten regions of the hollow blade is given in Figure 7. It is shown that the stiffness of P1-P5 and P6-P10 decreases gradually along the $Z$ direction (cf. Figure 1), and the stiffness of P1-P5 is larger than that of P6P10, respectively. According to equation (8), the higher the stiffness $k$ is, the lower the amplitude of the response function is. It is obvious that the machining vibrations are

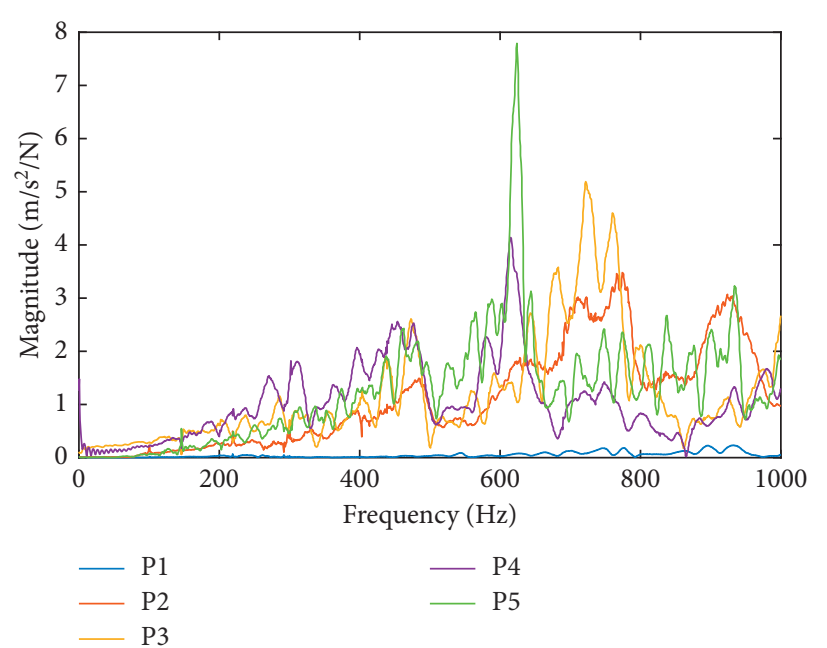

FIgURE 5: FRF curves of P1-P5 in the frequency domain.

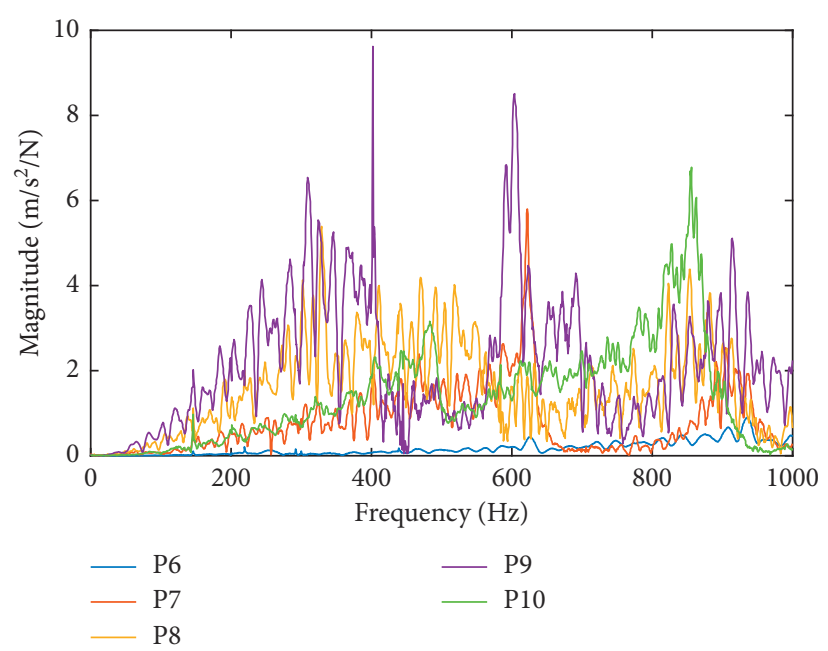

FIGURE 6: FRF curves of P6-P10 in the frequency domain.

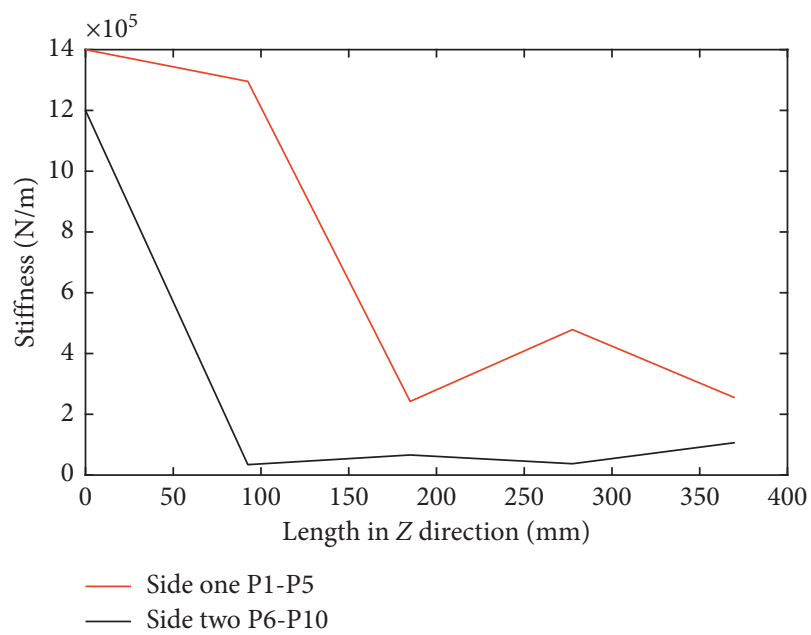

Figure 7: Computed stiffness of the hollow blade. 
easy to occur in the regions of the hollow blade near the right chuck. Besides, the stiffness of the hollow blade with a length of $100-350 \mathrm{~mm}$ in $Z$ direction is much weaker than other regions, which is largely affected by the overhang length of the blade and the fixed position.

It is apparent that the dynamic parameters of different regions on the hollow blade are different. To simplify the estimation of the machining stability of the hollow blade, an equivalent FRF curve was obtained by using an extremum method and a fitting method which are shown in Figures 8 and 9, respectively. Thus, the FRF curves of different regions on the hollow blade are equivalent to one curve. The equivalent FRF curve obtained by the fitting method locates between the peaks and valleys of the FRF curves. The magnitudes of the FRF curve obtained by the fitting method are considerably lower than those of weaker regions on the hollow blade, which would decrease the calculating accuracy of the dynamic parameters and the stability prediction. The extremum method prefers using the peaks of the FRF curves which is sensitive to the weak region of the hollow blade. The extremum method is to compare the FRF curves of different regions and achieve the equivalent FRF curve by choosing the maximum value in a certain frequency. The equivalent curves of P1-P5 and P6-P10 are presented in Figure 8. It is shown that the magnitude of the equivalent FRF curve obtained with the extremum method is larger than that of the fitting method. The frequencies corresponding to the amplitude of the equivalent FRF curves are the same for both the extremum method and the fitting method. A comparison of FRF curves shows that the equivalent FRF of P6-P10 is higher than that of P1-P5. The machining of the entire blade often requires a constant cutting parameter. Thus, the equivalent FRF curves of the hollow blade between P1 and P10 are obtained by the extremum method and the fitting method (cf. Figure 9).

The equivalent FRF curves obtained are substituted into equation (4) to compute the modal parameters of the machining system. Table 1 shows the computed modal parameters for the fitting method and the extremum method, respectively. It is shown that the modal parameters of regions P1-P5 with two methods are close. The first-order natural frequencies obtained with the fitting method and the extremum method are 300 and $370 \mathrm{~Hz}$, respectively, and the stiffnesses of two methods are $2.31 \times 10^{5} \mathrm{~N} / \mathrm{m}$ and $1.77 \times 10^{5} \mathrm{~N} / \mathrm{m} \mathrm{N} / \mathrm{m}$. The natural frequency of P6-P10 is considerably lower than that of P1-P5 at 268 and $286 \mathrm{~Hz}$, respectively. The first-order natural frequency and stiffness obtained by the extremum method are lower than those obtained with the fitting method. The first-order natural frequencies of the regions $\mathrm{P} 1-\mathrm{P} 10$, which are calculated by the extremum method and fitting method are $260 \mathrm{~Hz}$ and $308 \mathrm{~Hz}$. The stiffness achieved by the fitting method is $7.2 \times 10^{4} \mathrm{~N} / \mathrm{m}$ and the stiffness of the extremum method is $2.05 \times 10^{4} \mathrm{~N} / \mathrm{m}$. The stiffness of the extremum method is $71.5 \%$ smaller than that of the fitting method.

As illustrated in equation (9), the magnitude of the FRF is consistent with the magnitude of the blade and milling tool. The FRF curve of the milling tool is also measured by the impact test. Given that the magnitude of FRF is influenced by the angle $\beta$, the exciting and measuring directions on the tool were changed and the curve with smaller amplitude is shown in Figure 10. The FRF curve of the ballend milling cutter is compared with the equivalent FRF of the hollow blade obtained by the extremum method. It can be known from Figure 10 that the frequency response curve of the milling tool is considerably smaller than that of the blade. Therefore, the modal parameters of the tool are negligible in predicting the machining stability. From equation (9), it is known that the higher the magnitude of FRF, the lower the stiffness is. So, the stiffness of the milling tool is higher than that of the hollow blade. The stiffness of the hollow blade is the main contributor to the vibration.

\section{Stability Prediction and Experimental Verification}

According to SDM presented by Altintas [1], the singledegree stability prediction model is expressed as

$$
F(\omega)=\frac{1}{2} a K_{t}\left[A_{0}\right]\left[1-e^{-j \omega T}\right][\varnothing(j \omega)] F(\omega),
$$

where $\left[A_{0}\right]$ is the directional cutting coefficient matrix, $a$ is the depth of cut, $K_{t}$ is the tangential cutting force coefficient, and $[\varnothing(j \omega)]$ is the transfer function matrix. When the system is critically stable at the chatter frequency $\omega_{c}$, the root of the equation is formulated as follows:

$$
\begin{gathered}
\operatorname{det}\left[[I]-\frac{1}{2} a K_{t}\left[A_{0}\right]\left[1-e^{-j \omega_{c} T}\right]\left[\varnothing\left(j \omega_{c}\right)\right]\right]=0, \\
\Lambda=-\frac{N}{4 \pi} a K_{t}\left[A_{0}\right]\left[1-e^{-j \omega_{c} T}\right] .
\end{gathered}
$$

The critical axial depth of cut $a_{\lim }$ expressed as

$$
a_{\lim }=-\frac{2 \pi \Lambda_{R}}{\left(N K_{t}\right)\left(1+\left(\Lambda_{I}^{2} / \Lambda_{R}^{2}\right)\right)},
$$

where $\Lambda_{R}$ and $\Lambda_{I}$ stand for the real part and imaginary part of $\Lambda$. The SLD curve which contains the relationship between the critical axial depth of cut and the spindle speed can be obtained. For the differences of the dynamic parameters in ten regions, the SLD curves of ten regions are combined to be 3D SLD curves. The predicted 3D SLD curves for regions P1-P5 and P6-P10 are shown in Figures 11(a) and 11(b). The length in the figures represents the length of the blade along the $Z$ direction, where the total length of the blade is $350 \mathrm{~mm}$. The stiffness value $k$ is relatively large which allows predicting the machining stability with the high ultimate depth of cut. The maximum critical depths of cut for P1-P5 and $\mathrm{P} 6-\mathrm{P} 10$ are 0.94 and $2.4 \mathrm{~mm}$, respectively. The middle positions of the blade such as at $\mathrm{P} 6, \mathrm{P} 7$, and $\mathrm{P} 8$ have a low rigidity which results in a small critical depth of cut.

The 3D SLD can predict the stability of different regions accurately. However, it will take too much time for the calculation of 3D SLD and the chatter will occur in the region where the critical axial depth of cut is the least. To improve the calculation efficiency, 2D SLD curves of the fitting method and extremum method are illustrated in 


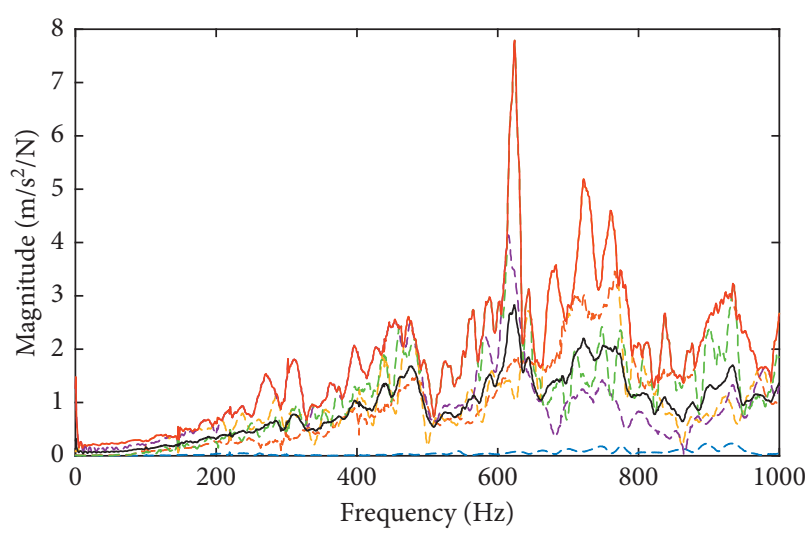

P1-P5 fitting method _ P1-P5 extremum method

(a)

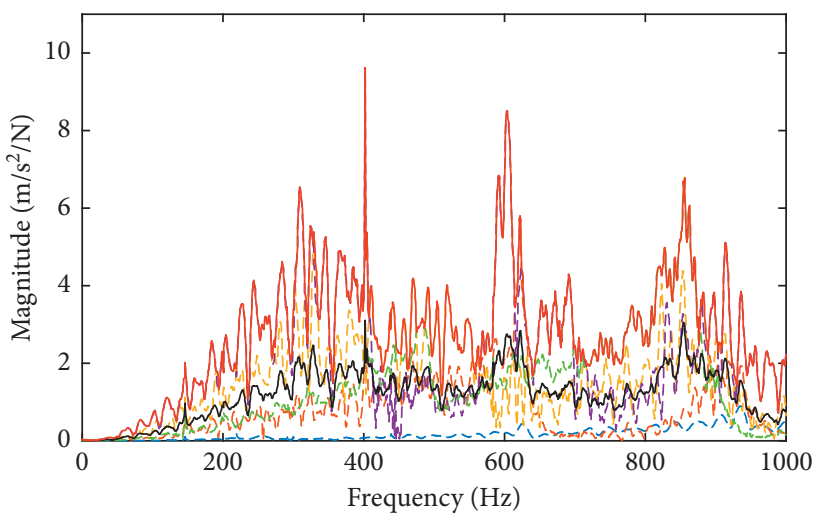

P6-P10 fitting method

- P6-P10 extremum method

(b)

Figure 8: Comparison between the fitting and extremum methods of different regions. (a) Regions 1-5 (P1-P5). (b) Regions 6-10 (P6-P10).

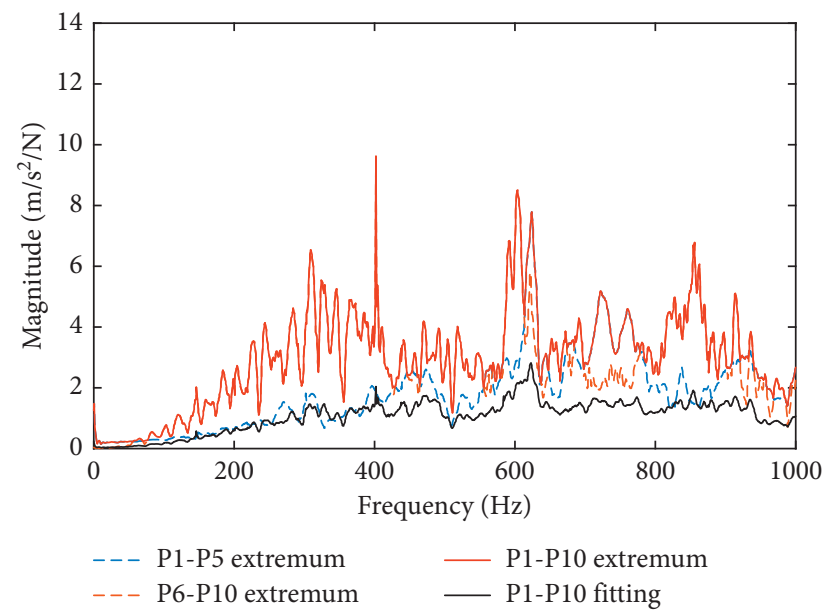

FIGURE 9: Equivalent FRF curves for P1-P10 obtained by the extremum method and fitting method.

TABle 1: Dynamic parameters with two methods.

\begin{tabular}{lccc}
\hline Method & First-order frequency $(\mathrm{Hz})$ & Damping ratio & Stiffness $(\mathrm{N} / \mathrm{m})$ \\
\hline Fitting method (P1-P5) & 300 & 0.4289 & 231,730 \\
Extremum method (P1-P5) & 370 & 0.3219 & 177,240 \\
Fitting method (P6-P10) & 286 & 0.4133 & 64,128 \\
Extremum method (P6-P10) & 268 & 0.3994 & 25,598 \\
Fitting method (P1-P10) & 308 & 0.4037 & 72,007 \\
Extremum method (P1-P10) & 260 & 0.3061 & 20,533 \\
\hline
\end{tabular}

Figure 12. The influence of the extremum and fitting methods on the stability prediction is subsequently compared. The SLD curves obtained from the extremum method on P1-P5, P6-P10, and P1-P10 and the fitting method on $\mathrm{P} 1-\mathrm{P} 5$ and P6-P10 are analyzed (cf. Figure 12). The predicted result of the stability on regions P1-P10 with the fitting method is higher than that of the extremum method. A comparison between the two methods shows similar results for P6-P10 and P1-P5. Figure 12 shows that the stiffness of P6-P10 is lower than that for P1-P5, and the SLD curve for P1-P10 has the smallest stable region.

The cutting tests are carried out to verify the predicting results of stability on a Mazak 200 IV ST turn-milling center. A ball-end milling tool with a diameter $D$ of $10 \mathrm{~mm}$ and a teeth number $\mathrm{N}$ of 2 is employed. The radial depth of cut of $0.2 \mathrm{~mm}$, the feed rate of $500 \mathrm{~mm} / \mathrm{min}$, and the angle $\beta$ of $45^{\circ}$ are used as the machining parameters. The reasonable axial cutting depth and spindle speed are selected to verify SLD 


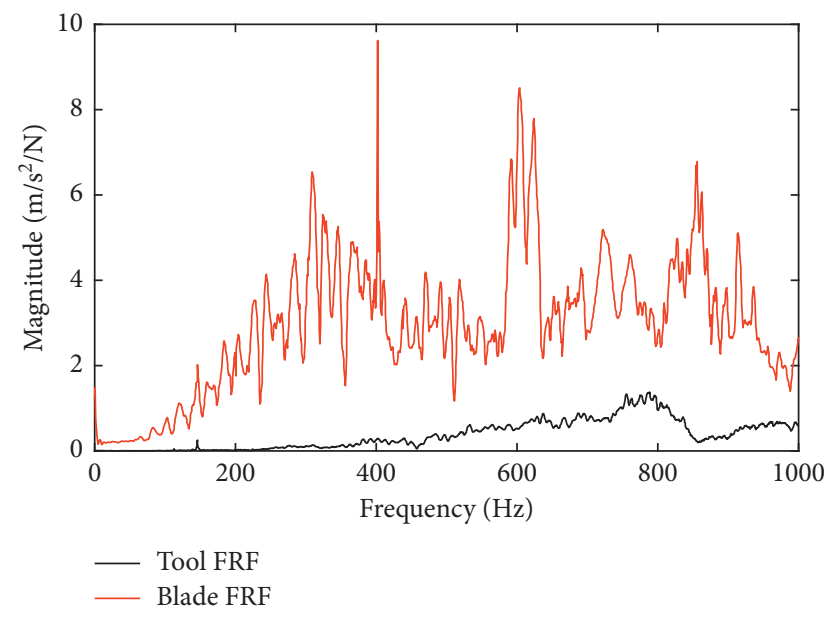

FIgURE 10: Comparison of FRF between the tool and the blade.

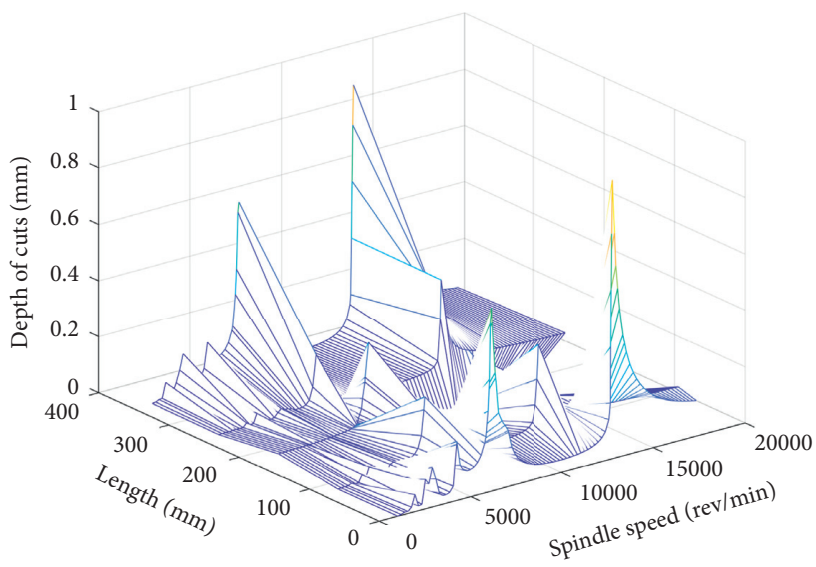

(a)

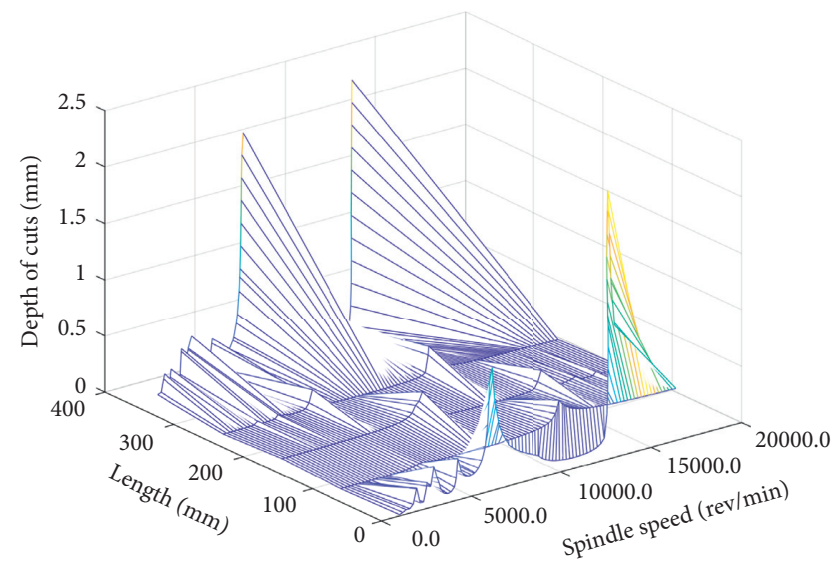

(b)

Figure 11: Predicted 3D SLDs for P1-P5 and P6-P10. (a) Predicted 3D SLD for P1-P5. (b) Predicted 3D SLD for P6-P10.

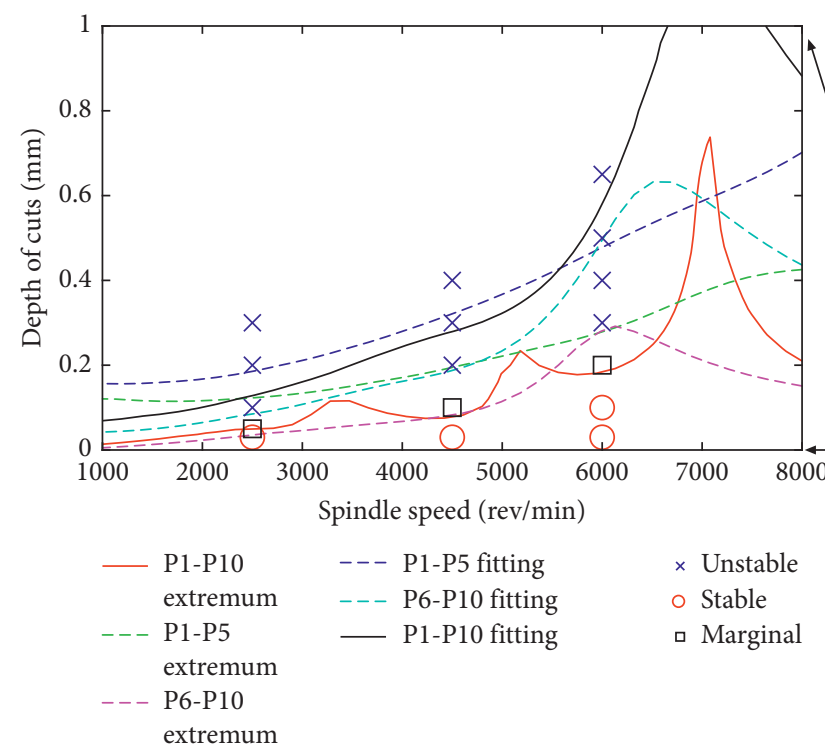

(a)

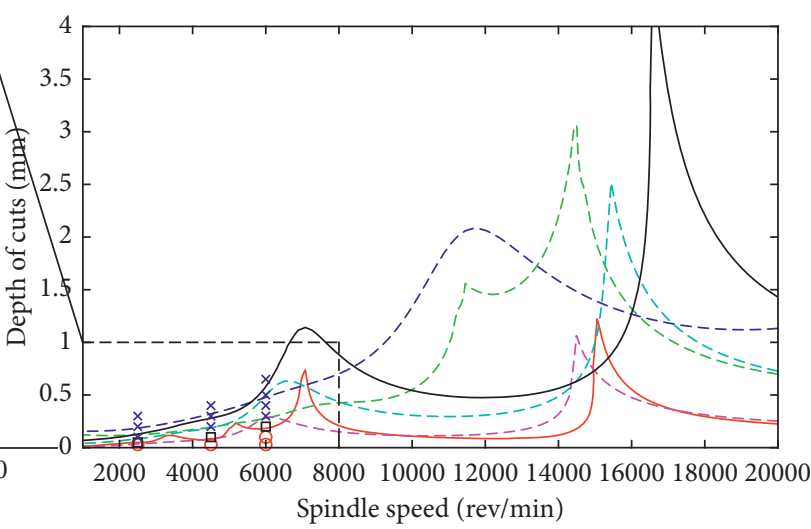

$\begin{aligned}- & \text { P1-P10 } \\ & \text { extremum } \\ --- & \text { P1-P5 } \\ & \text { extremum } \\ --- & \text { P6-P10 } \\ & \text { extremum }\end{aligned}$

- - - P1-P5 fitting

$\times$ Unstable

- Stable

--- P6-P10 fitting

a Marginal

FIGURE 12: Predicted SLD for the fitting method and extremum method. 


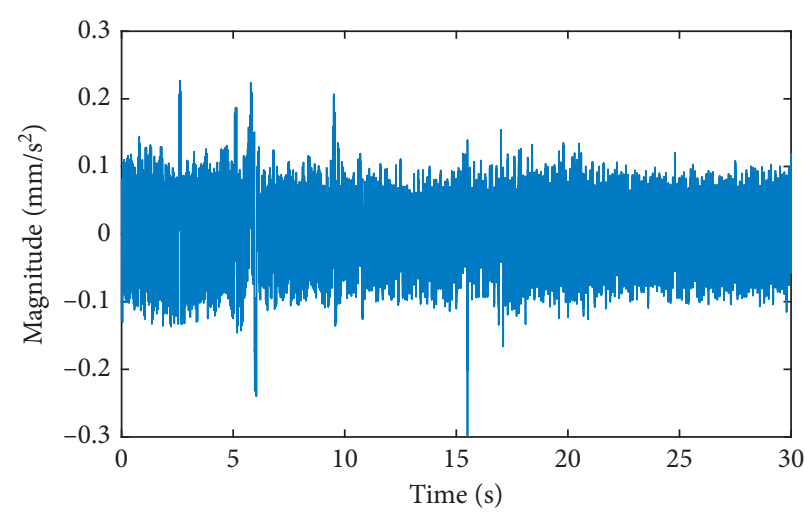

(a)

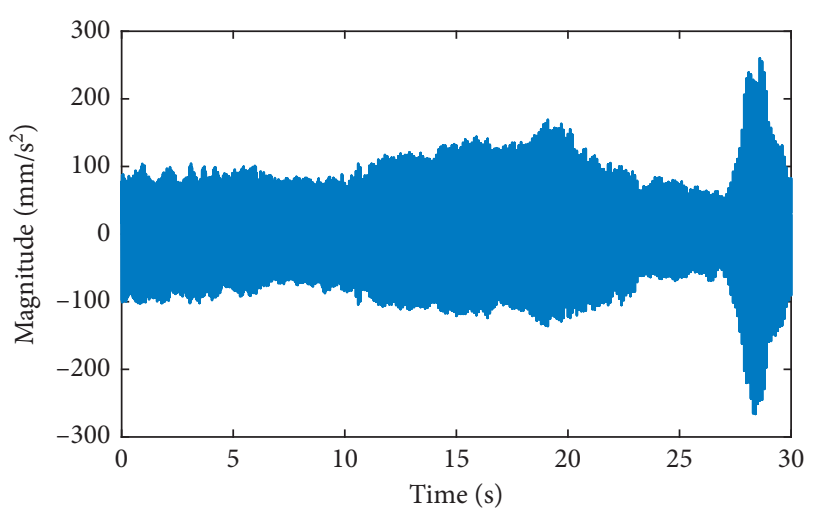

(b)

Figure 13: Acceleration signal analysis of the hollow blade in the machining process. (a) Stable machining (time domain). (b) Unstable machining (time domain).

and the spindle speed is in the range of $0-8,000 \mathrm{rpm}$. In order to confirm the reliability of SLD, the machining experiments with axial cutting depths of $0.03 \mathrm{~mm}, 0.1 \mathrm{~mm}$, $0.2 \mathrm{~mm}, 0.3 \mathrm{~mm}, 0.4 \mathrm{~mm}, 0.5 \mathrm{~mm}, 0.6 \mathrm{~mm}$ are implemented when the spindle speed is $6,000 \mathrm{rpm}$. And the axial cutting depth is also set as $0.03 \mathrm{~mm}, 0.05 \mathrm{~mm}, 0.1 \mathrm{~mm}, 0.2 \mathrm{~mm}$, and $0.3 \mathrm{~mm}$ when the spindle speed is $2,500 \mathrm{rpm}$. It can be known from the experiments that the axial cutting depth of $0.2 \mathrm{~mm}$ is the marginal zone in SLD. The machining process is unstable when the spindle speed is $6,000 \mathrm{rpm}$ and axial cutting depth is higher than $0.2 \mathrm{~mm}$. The opposite result will be received when the axial cutting depth is lower than $0.2 \mathrm{~mm}$. The similar results can be achieved that the critical depth of cut is $0.05 \mathrm{~mm}$ when the spindle speed is $2,500 \mathrm{rpm}$. Then, the parameters of spindle speeds of 2,500 and $6,000 \mathrm{rpm}$ and the axial depth of cut of 0.3 and $0.03 \mathrm{~mm}$ are selected to verify that the extremum method is more reliable than the fitting method. When the spindle speed is $2,500 \mathrm{rpm}$, the prediction results of the extremum method and fitting method are both stable at the axial cutting depth of $0.03 \mathrm{~mm}$, the prediction results of both methods are unstable at the axial cutting depth of $0.3 \mathrm{~mm}$. The prediction results are considered to be different at the axial depth of $0.3 \mathrm{~mm}$ when the spindle speed is $6,000 \mathrm{rpm}$. The fitting method predicts that it is in a stable state, while the extremum method considers that the parameter is above the marginal of SLD, and the result of prediction is unstable. The conclusion can be collected that the predicting result of the extremum method is more accurate than the fitting method by comparison of SLD. Figure 13(a) shows the acceleration signal in the stable zone as collected with an acceleration sensor. The time domain acceleration signal for the unstable zone is also acquired and shown in Figure 13(b). The signals are compared when the axial depth of cut is $0.03 \mathrm{~mm}$ and $0.3 \mathrm{~mm}$ and the spindle speeds are $6000 \mathrm{rpm}$. It can be observed from Figure 13(b) that the acceleration magnitudes are higher than that of Figure 13(a) and the cutting process became unstable.

\section{Conclusions}

In order to avoid the chatter in turn-milling of hollow blades with HCSs, this paper presents a measurement-based approach for modal analysis and stability prediction of the turn-milling process of the hollow blades with HCSs. The FRF curves on the blade and the cutting tool were achieved by the impact test and were approximated as an equivalent FRF curve by using an extremum method, which is compared with the result by using a fitting method. The dynamic parameters involving the natural frequency, damping ratio, and stiffness are calculated based on the equivalent FRF curve and the dynamic model. The 2D and 3D stability lobe diagrams were drawn with the semidiscretization method to predict the machining stability of the hollow blade. The cutting tests were performed to validate the feasibility of the proposed approach. The results show that the equivalent FRF curve obtained by the extremum method is larger than that of the fitting method in the range of $0-1000 \mathrm{~Hz}$. The acceleration signals with spindle speeds of 2,500 and $6,000 \mathrm{rpm}$ and axial depth of cuts of 0.3 and $0.03 \mathrm{~mm}$ are analyzed. When the spindle speed is $2500 \mathrm{rpm}$, the predicting results of the fitting and extremum method are almost the same. When the axial depth of cut is $0.3 \mathrm{~mm}$ and the spindle speed is $6000 \mathrm{rpm}$, the predicting result of the extremum method is more consistent with the test result than that of the fitting method, which proves that the stability prediction using the extremum method is more reliable.

\section{Data Availability}

All the data regarding this paper are available, and the author can provide them if they are required.

\section{Conflicts of Interest}

The authors declare that they have no conflicts of interest. 


\section{Acknowledgments}

This work was partially supported by National Natural Science Foundation of China (no. 51805258), Natural Science Foundation of Jiangsu Province (no. BK20180441), Fundamental Research Funds for Central Universities (NZ2016107), the National Science Foundation for PostDoctoral Scientists of China (no. 2019M661824), the University Science Research Project of Jiangsu Province (18KJA460004), and Jiangsu Key Laboratory of Precision and Micro-Manufacturing Technology.

\section{References}

[1] M. Wan, X.-B. Dang, W.-H. Zhang, and Y. Yang, "Optimization and improvement of stable processing condition by attaching additional masses for milling of thin-walled workpiece," Mechanical Systems and Signal Processing, vol. 103, pp. 196-215, 2018.

[2] Z. Zhang, H. Li, X. Liu, W. Zhang, and G. Meng, "Chatter mitigation for the milling of thin-walled workpiece," International Journal of Mechanical Sciences, vol. 138-139, pp. 262-271, 2018.

[3] M. A. Butt, Y. Yang, X. Pei, and Q. Liu, "Five-axis milling vibration attenuation of freeform thin-walled part by eddy current damping," Precision Engineering, vol. 51, pp. 682-690, 2018.

[4] W. Han, K. Zhang, and G. Wang, "Superplastic forming and diffusion bonding for honeycomb structure of Ti-6Al-4V alloy," Journal of Materials Processing Technology, vol. 183, no. 2-3, pp. 450-454, 2007.

[5] M. Wan, T.-Q. Gao, J. Feng, and W. H. Zhang, "On improving chatter stability of thin-wall milling by prestressing," Journal of Materials Processing Technology, vol. 264, pp. 32-44, 2019.

[6] M. Wan, J. Feng, Y.-C. Ma, and W.-H. Zhang, "Identification of milling process damping using operational modal analysis," International Journal of Machine Tools and Manufacture, vol. 122, pp. 120-131, 2017.

[7] O. B. Adetoro, W. M. Sim, and P. H. Wen, "An improved prediction of stability lobes using nonlinear thin wall dynamics," Journal of Materials Processing Technology, vol. 210, no. 6-7, pp. 969-979, 2010.

[8] S. Seguy, G. Dessein, and L. Arnaud, "Surface roughness variation of thin wall milling, related to modal interactions," International Journal of Machine Tools and Manufacture, vol. 48, no. 3-4, pp. 261-274, 2008.

[9] G. Stepan, A. K. Kiss, B. Ghalamchi, J. Sopanen, and D. Bachrathy, "Chatter avoidance in cutting highly flexible workpieces," CIRP Annals-Manufacturing Technology, vol. 66, pp. 377-380, 2017.

[10] E. Budak, L. T. Tunç, S. Alan, and H. N. Özgüven, "Prediction of workpiece dynamics and its effects on chatter stability in milling," CIRP Annals, vol. 61, no. 1, pp. 339-342, 2012.

[11] Y. Ding and L. Zhu, "Investigation on chatter stability of thinwalled parts considering its flexibility based on finite element analysis," The International Journal of Advanced Manufacturing Technology, vol. 94, no. 9-12, pp. 3173-3187, 2018.

[12] X. Jin, Y. Sun, Q. Guo, and D. Guo, “3D stability lobe considering the helix angle effect in thin-wall milling," The International Journal of Advanced Manufacturing Technology, vol. 82, no. 9-12, pp. 2123-2136, 2016.
[13] Y. Altinta and E. Budak, "Analytical prediction of stability lobes in milling," CIRP Annals, vol. 44, no. 1, pp. 357-362, 1995.

[14] X. Liu, Y. Li, and G. Chen, "Multimode tool tip dynamics prediction based on transfer learning," Robotics and Computer-Integrated Manufacturing, vol. 57, pp. 146-154, 2019.

[15] C. Sun and Y. Altintas, "Chatter free tool orientations in 5-axis ball-end milling," International Journal of Machine Tools and Manufacture, vol. 106, pp. 89-97, 2016.

[16] Q. Song, Z. Liu, Y. Wan, G. Ju, and J. Shi, "Application of Sherman-Morrison-Woodbury formulas in instantaneous dynamic of peripheral milling for thin-walled component," International Journal of Mechanical Sciences, vol. 96-97, pp. 79-90, 2015.

[17] E. Budak and Y. Altintas, "Analytical prediction of chatter stability in milling-Part I: general formulation," Journal of Dynamic Systems, Measurement, and Control, vol. 120, no. 1, pp. 22-30, 1998.

[18] T. Insperger and G. Stépán, "Updated semi-discretization method for periodic delay-differential equations with discrete delay," International Journal for Numerical Methods in Engineering, vol. 61, no. 1, pp. 117-141, 2004.

[19] C. Henninger and P. Eberhard, "Improving the computational efficiency and accuracy of the semi-discretization method for periodic delay-differential equations," European Journal of Mechanics-A Solids, vol. 27, no. 6, pp. 975-985, 2008.

[20] X. Zhou, D. Zhang, M. Luo et al., "Tool path dependent chatter suppression in multi-axis milling of hollow fan blades with ball-end cutter," The International Journal of Advanced Manufacturing Technology, vol. 72, no. 5-8, pp. 643-651, 2014.

[21] Y. Altintas, Manufacturing Automation: Metal Cutting Mechanics, Machine Tool Vibrations, and CNC Design, Cambridge University Press, Cambridge, UK, 2012. 